University of Nebraska - Lincoln

DigitalCommons@University of Nebraska - Lincoln

Sociology Department, Faculty Publications

Sociology, Department of

2020

\title{
A comparison of frequency of alcohol and marijuana use using short message service surveying and survey questionnaires among homeless youth
}

\author{
Kimberly A. Tyler \\ University of Nebraska - Lincoln, kim@ktresearch.net \\ Kristen M. Olson \\ University of Nebraska-Lincoln, kolson5@unl.edu
}

Follow this and additional works at: https://digitalcommons.unl.edu/sociologyfacpub

Part of the Family, Life Course, and Society Commons, and the Social Psychology and Interaction

Commons

Tyler, Kimberly A. and Olson, Kristen M., "A comparison of frequency of alcohol and marijuana use using short message service surveying and survey questionnaires among homeless youth" (2020). Sociology Department, Faculty Publications. 741.

https://digitalcommons.unl.edu/sociologyfacpub/741

This Article is brought to you for free and open access by the Sociology, Department of at DigitalCommons@University of Nebraska - Lincoln. It has been accepted for inclusion in Sociology Department, Faculty Publications by an authorized administrator of DigitalCommons@University of Nebraska - Lincoln. 


\title{
A comparison of frequency of alcohol and marijuana use using short message service surveying and survey questionnaires among homeless youth
}

\author{
Kimberly A. Tyler and Kristen Olson \\ Department of Sociology, University of Nebraska-Lincoln, Lincoln, NE, USA \\ Correspondence - Kimberly A. Tyler kim@ktresearch.net Department of Sociology, \\ University of Nebraska-Lincoln, 717 Oldfather Hall, Lincoln, NE, 68588-0324
}

\begin{abstract}
Background: There are several benefits to using short message service surveying (SMS) to gather data on substance use from homeless youth, including capturing data "in the moment" and verifying the timing of one behavior relative to another. Though SMS is a valuable data collection tool with highly mobile populations that otherwise are difficult to longitudinally sample, the reliability of SMS compared with surveys is largely unknown with homeless youth. Examining the reliability of SMS is important because these data can provide a more nuanced understanding of the relationships between various risk behaviors, which may lead to better intervention strategies with these youth.

Objectives: We compared past 30-day survey and SMS data for youth's alcohol and marijuana use.

Methods: Interviewed 150 homeless youth (51\% female) using surveys and SMS. Results: Past 30-day survey and SMS data revealed moderately strong correlations for alcohol $\left(r_{\mathrm{s}}=.563\right)$ and marijuana $\left(r_{\mathrm{s}}=.564\right)$. Regression analysis revealed that
\end{abstract}

Published in The American Journal of Drug and Alcohol Abuse 2020, VOL. 46, NO. 4, 401-407 doi:10.1080/00952990.2019.1680682

Copyright (C) 2019 Taylor \& Francis Group, LLC

Submitted 29 April 2019; revised 28 September 2019; accepted 10 October 2019;

published 3 December 2019. 
independent variables were similarly associated with alcohol and marijuana use when comparing survey and SMS data with two exceptions: heterosexual youth reported less alcohol use in SMS data compared to survey data $(\beta=-.212 ; p<.05$ vs. $\beta=-.006 ; p>.05$, respectively) and youth whose parents had alcohol problems reported less marijuana use in survey data compared to SMS data $(\beta=-.277 ; p<.01$ vs. $\beta=-.150 ; p>.05$, respectively).

Conclusion: Findings indicate SMS and surveys are both reliable methods of gathering data from homeless youth on substance use.

Keywords: Alcohol, marijuana, homeless youth, short message service, survey questionnaires

\section{Introduction}

Research finds that homeless youth are 2-3 times more likely to use alcohol and marijuana compared to housed youth $(1,2)$. Moreover, $75 \%$ of homeless youth report lifetime alcohol and/or marijuana use $(3,4)$ whereas past 30-day prevalence rates for alcohol and marijuana usage have been found to be $68 \%$ and $66 \%$, respectively (5). Lim, Rice, and Rhoades (6),found that marijuana is the drug used by homeless youth most frequently (73\%) followed by alcohol (69\%). More recently, researchers have begun to use short message service surveying (SMS) with homeless youth to collect substance use data. For example, Santa Maria et al. (7), found that 40 out of 66 homeless youth reported using drugs on at least one day in the prior 21 days and of these 40 youth, 36 of them used marijuana, on average, a total of 5 days.

Although a couple of recent studies have used SMS to gather data on homeless youth's substance use $(7,8)$, the vast majority of research in this area has used survey questionnaires to collect such data (4). There are several benefits, however, to using daily SMS data collection through ecological momentary assessment (EMA). First, EMA allows researchers to capture data "in the moment" about an individual's current behavior in their natural environment (9). Second, unlike the use of survey questionnaires, which are generally retrospective and cross-sectional in nature, EMA via SMS surveying verifies the timing of one behavior relative to another, allowing for temporal sequencing (10) and minimizes recall biases (11). Specifically, using SMS to collect daily data from this group of youth allows researchers to verify the time ordering between various risky behaviors such as substance use and victimization for example, and knowing the ordering of events is important for intervention 
and prevention strategies with this population. Third, the multitude of data points generated with SMS allows researchers to examine various patterns of behavior over time such as how youths' alcohol use fluctuates over 30 days, with which specific events, and the time ordering of these events. Finally, because EMA via SMS allows researchers to collect data "in the moment" and verify the timing of one behavior relative to another, SMS is a valuable tool for data collection with a highly mobile population that would otherwise be difficult to sample on a routine basis (12).

Though there are many benefits to using SMS to collect daily data from homeless youth, there are few studies that have done so. As such, there is limited research on whether SMS yields reliable data with this specific population. In other words, does gathering data within the same time frame but with different methods yield similar results? Researchers examining a population of substance using men who have sex with men found that alcohol use and methamphetamine use were reported more often with daily EMA texts compared to retrospective survey data suggesting that EMA methods may provide a more complete picture of substance use among this specific population (13). As such, the purpose of the current study is three-fold. First, we compare follow-up survey questionnaire data and SMS data to examine past 30-day alcohol use and past 30-day marijuana use. Second, we examine how similar actual reports of past 30-day ever drinking alcohol and ever using marijuana are for the follow-up survey and SMS data. Third, we examine whether demographic characteristics and early family risk factors are similarly associated with past 30-day alcohol and marijuana use for both follow-up survey and SMS data. Examining the reliability of SMS is important because these data can provide a more nuanced understanding of the relationships between various risk behaviors beyond that collected with traditional methods, which may lead to better prevention and intervention strategies with this group of youth.

\section{Method}

Data are from the Homeless Youth Texting Project, a pilot study designed to examine risk and protective factors for substance use and to field test ecological momentary assessment (EMA) via SMS to ascertain its utility 
and feasibility with homeless youth. Findings from the feasibility study are reported elsewhere (Tyler \& Olson, 14). From August 2014 through October 2015, 150 homeless youth were interviewed in two Midwestern cities. Of the 150 respondents interviewed at baseline, 112 youth or 75\% completed a follow-up interview. The Institutional Review Board at the University of Nebraska-Lincoln approved this study.

Eligibility required youth to be between 16 and 22 years of age and homeless or runaway. Homeless youth, as inclusively defined by the 2015 reauthorization of the McKinney-Vento Homeless Assistance Act, includes those who lack permanent housing such as spending the previous night in a shelter, public place, on the street, with friends, or in a transitional facility, or other places not intended as a domicile (15). Runaway includes those under age 18 who spent the previous night away from home without parental permission (16). Participants were recruited through three local agencies which offer various services such as emergency shelter and food programs.

Four trained and experienced interviewers conducted the interviews. Interviewers approached youth at shelters, food programs, and during street outreach. Informed consent was obtained from youth, who were told that the study had three parts and if they agreed to participate, they would need to complete a baseline structured interview, the SMS portion, and a follow-up, structured interview. The two interviews, which were conducted in shelter interview rooms, local library, or outside (weather permitting) lasted 45 minutes and 15 minutes, respectively. Participants received a $\$ 20$ and $\$ 10$ gift card to a local store for completing the baseline and follow-up interview, respectively. Less than $3 \%$ of youth $(\mathrm{N}=5)$ refused to participate or were ineligible. The current study reports results from the baseline interview, the SMS portion, and the follow-up interview.

\section{Cell phone distribution}

Upon completing the baseline interview, participants were given a disposable cell phone and told they would receive 11 texts per day over the next 28-30 days and then would be re-contacted in approximately 30 days for a follow-up interview. The blocks of texts came at 10:00 am, 4:00 pm and 9:30 pm. Text questions were sent from an automated system, set up to send out text questions in the same order and at the same 
time each day. Responding to each text question required participants to enter a number(s). Typically, 3-4 days prior to the end of their texting period, youth were sent a text informing them how many texting days were left and to set up a follow-up interview. Those who responded to every text question (11 texts per day) were paid $\$ 50$ cash (prorated at $\$ 0.14$ per response) and those who responded to at least $85 \%$ of texts also received a bonus $\$ 10$ gift card.

\section{Measures}

We use both SMS data and follow-up survey questionnaire data to compare the reliability of these two different data collection methods.

\section{Text questions}

From the text (SMS) data, we use two questions that were asked at 9:30 pm: (1) alcohol use: "how many drinks tonight" (range 0-7 or more drinks) and (2) marijuana use: "used any of these drugs tonight" (weed, crank, meth, coke, inhalant, heroin, ecstasy, other, none). From this list of drugs, we examine marijuana (i.e. weed) for the current analyses. We summed these indicator variables across all of the days for which there were texting data available for each respondent. Thus, each variable represents a count of the number of days during the texting period that the respondent used either alcohol or marijuana. For additional analyses, we also created two dichotomized variables which represent whether the respondent had "ever" used alcohol ( $1=$ any drinks, $0=$ no drinks) and "ever" used marijuana ( 1 = used marijuana, $0=$ did not use marijuana) in the past 30 days.

\section{Survey questions}

From the follow-up survey questionnaire data, which examines the same time frame as the SMS data, we include measures of alcohol and marijuana, which asked youth about their usage during the past 30 days $(0=$ never, $1=1-2$ times, $2=3-4$ times, $3=5-7$ times, $4=8-10$ times, $5=$ more than 10 times). Alcohol included three items (beer, wine, or hard liquor), which were combined into a mean scale and marijuana was a single item indicator. We also dichotomized the past 1-month reports into $1=$ any use and $0=$ no use of each substance. 
The following measures are from the baseline survey questionnaire data and are used in the multivariate analyses, described below.

Child physical abuse was a summed scale of 16 items from the Conflict Tactics Scale (17). Youth were asked, for example, how frequently their parent/caretaker shook them or kicked them hard $(0=$ never to 6 $=$ more than 20 times). A mean scale was created where a higher score indicated more physical abuse $(\alpha=.93)$.

Child sexual abuse included seven items [adapted from 18] that asked youth how often any adult or someone at least five years older than them asked them, for example, to do something sexual or had them touch the adult sexually ( $0=$ never to $6=$ more than 20 times). Due to skewness, the seven items were first dichotomized ( $0=$ never and $1=$ at least once) and then a count variable was created where a higher score equaled a greater number of different types of sexual abuse experienced $(\alpha=.92)$. These same items have been used in prior studies of homeless youth [18, $\alpha=.93 ; 19, \alpha=.88]$.

Parental alcohol problems and parental drug problems [adapted from the CAST-6: 20] each consisted of three items that asked respondents, for example, if they ever thought that their parent had an alcohol/drug problem and if they ever encouraged their parent to quit using alcohol/ drugs ( $0=$ no; 1 = yes). Due to skewness, both summed variables were dichotomized where 0 = no parental alcohol problem; 1 = parent had alcohol problem and $0=$ no parental drug problem; 1 = parent had a drug problem, respectively.

Gender was coded 0 = male; 1 = female. Sexual orientation was coded 0 = lesbian, gay, bisexual (LGB); 1 = straight or heterosexual. Age was a continuous variable that asked youth their current age at the time of the interview.

\section{Statistical analysis}

First, we compared the follow-up survey and SMS data to examine past 30-day alcohol use and past 30-day marijuana use using Spearman's rank-order correlations. Second, we looked at the agreement between youth reports of "ever" using alcohol and "ever" using marijuana comparing the follow-up survey data and SMS data using crosstabs, kappa, and chi square statistics. Third, we used ordinary least squares (OLS) regression to examine demographic characteristics and early family risk 
factors and compared their association with past 30-day alcohol and past 30-day marijuana use using the follow-up survey data and SMS data. All analyses were conducted using SPSS version 25.

We aggregate all of the data to the youth-level. The sample size for each set of analyses varies due to missing data, mostly from missing texting data. We have an average of 13.99 days of data with usable texting reports on drinking from the youth and an average of 15.18 days with usable texting data on marijuana use. Overall, 144 youth reported information about drinking, with a mean of 1.05 days with drinking over the time period, with drinking on an average across youth of $9.2 \%$ of the days for which we have texting data. For marijuana use, 140 youth reported using marijuana for an average of 2.44 days over the time period, an average across youth of $15.5 \%$ of the days for which we have texting data.

\section{Results}

\section{Sample characteristics}

Demographic information based on the baseline survey questionnaire data included 150 homeless youth ages 16 to 22 years $(M=19.4$ years $)$. One-half (51\%) were female, and $22 \%$ identified as LGB. $98.6 \%$ of youth experienced at least one form of child physical abuse and $41 \%$ experienced one or more forms of child sexual abuse. Parental substance use was also problematic: $43 \%$ of youth reported that a parent had at least one problem with alcohol and almost $42 \%$ of young people said a parent had one or more problems with drugs.

\section{Comparison of survey and SMS data}

Table 1 shows the correlation matrix comparing past 30-day alcohol and marijuana use using both the follow- up survey data and SMS data. Results revealed moderate strength correlations between past 30-day alcohol use $\left(r_{s}=.563 ; p<.01\right)$ and past 30 -day marijuana use $\left(r_{s}=.564\right.$; $p<.01$ ) using the SMS and followup survey data. The correlations were moderate in strength, reflecting both differential response scales and some discrepancy in the exact number of days of reported use across the two different data collection methods. 
Table 1. Correlation matrix comparing follow-up survey and SMS data for past 30-day alcohol and marijuana use.

\begin{tabular}{llllll} 
& 1 & 2 & 3 & 4 \\
\hline 1 & Past 30-day alcohol use (SMS) & - & & & \\
2 & Past 30-day alcohol use (follow-up survey) & $.563^{* *}$ & - & & \\
3 & Past 30-day marj. use (SMS) & $.594^{* *}$ & $.404^{* *}$ & - & \\
4 & Past 30-day marj. use (follow-up survey) & $.495^{* *}$ & $.493^{* *}$ & $.564^{* *}$ & - \\
\hline
\end{tabular}

Marj $=$ marijuana

$\mathrm{n}=106 .{ }^{* *} p<.01 ;{ }^{*} p<.05$

To facilitate comparisons across the two data collection methods, we dichotomized the alcohol and marijuana reports in both the texting and follow-up survey data into measures of "ever" versus "never" using alcohol or marijuana during the past 30 days. Among the 144 youth who completed at least one day with texting data on alcohol use, $42.4 \%$ reported using alcohol at least once over the texting period. Among the 111 youth who completed the survey, 44.1\% reported using alcohol during the last 30 days. Among the 140 youth who completed at least one day with texting data on using marijuana, 39.3\% reported using marijuana. Among the 111 youth with survey data, 37.8\% reported using marijuana at least once during the last 30 days. It is important to note that these are very similar estimates for both alcohol and marijuana, and in fact, were not statistically different from each other for either substance when the estimates were examined on the same set of respondents (alcohol, $\mathrm{t}(109)=1.35, p=.18$; marijuana, $\mathrm{t}(105)=0.20, p=.84)$.

We have data for both sets of measures from 110 youth for alcohol and from 106 youth for marijuana. The two reports agree 75.5\% of the time for alcohol (kappa $=0.49$ ) and $76.4 \%$ of the time for marijuana (kappa $=0.50)$. Table 2 shows the crosstabulation between the two measures dichotomized to "ever" versus "never" reports during the last 30 days $\left(\chi^{2}=27.17, p<.0001\right)$. Among those who reported never using alcohol during the last 30 days, $83.9 \%$ also never reported using alcohol via SMS, but 16.1\% reported using alcohol at least once via SMS. Among those who reported using alcohol via the survey data, 64.6\% also reported using alcohol during at least one day of the SMS data, and $35.4 \%$ did not report using alcohol via SMS. The patterns and estimates for marijuana are almost identical. 
Table 2. Crosstabulations between alcohol $(n=110)$ and marijuana $(n=106)$ use for follow-up (f/u) survey and SMS data.

\begin{tabular}{lcccc} 
& \multicolumn{4}{c}{ Ever Past 30-day alcohol use (SMS) } \\
& No & Yes & Total & $\chi^{2}$ \\
\hline Ever past 30-day alcohol use (f/u survey) & $\mathrm{N}(\%)$ & $\mathrm{N}(\%)$ & $\mathrm{N}(\%)$ & \multirow{2}{*}{$27.17^{* * *}$} \\
No & $52(83.9)$ & $10(16.1)$ & $62(100)$ & \\
Yes & $17(35.4)$ & $31(64.6)$ & $48(100)$ & \\
Total & $69(62.7)$ & $41(37.3)$ & $110(100)$ & \\
kappa & 0.49 & & & \\
\hline
\end{tabular}

Ever Past 30-day marijuana use (SMS)

\begin{tabular}{lcccc} 
& No & Yes & Total & $\chi^{2}$ \\
\hline Ever past 30-day marj. use (f/u survey) & $\mathrm{N}(\%)$ & $\mathrm{N}(\%)$ & $\mathrm{N}(\%)$ & $27.05^{* * *}$ \\
No & $52(81.3)$ & $12(18.8)$ & $64(100)$ & \\
Yes & $13(31.0)$ & $29(69.0)$ & $42(100)$ & \\
Total & $65(61.3)$ & $41(38.7)$ & $106(100)$ & \\
kappa & 0.50 & & & \\
\hline
\end{tabular}

SMS $=$ short message service. Marj = marijuana.

$* * * p<.0001$

Part of this difference, however, may be explained by the missing data for youth responding to the SMS questions. We have fewer days of SMS data for the youth who reported using alcohol in the follow-up survey and not in the SMS data (12.29 days), compared to 17.61 days for those who reported using in both data collections, 15.94 days for those who reported not using in both data collections, and 17.5 days for those who reported not using in the follow-up survey, but did report using in the SMS data. The differences in the number of days with usable data for marijuana are more striking - we have only 10.23 days of texting data for those who reported using marijuana in the follow-up survey, but not via the SMS data, compared to 18 to 20 days for all other groups. Thus, it is likely that youth were using alcohol and/or marijuana on days for which we do not have texting data.

\section{Multivariate results}

We estimated ordinary least squares regression models (Table 3) to examine whether demographic characteristics and early family risk factors were similarly associated with past 30-day alcohol and marijuana use for both the follow-up survey data and SMS data. Results for alcohol 


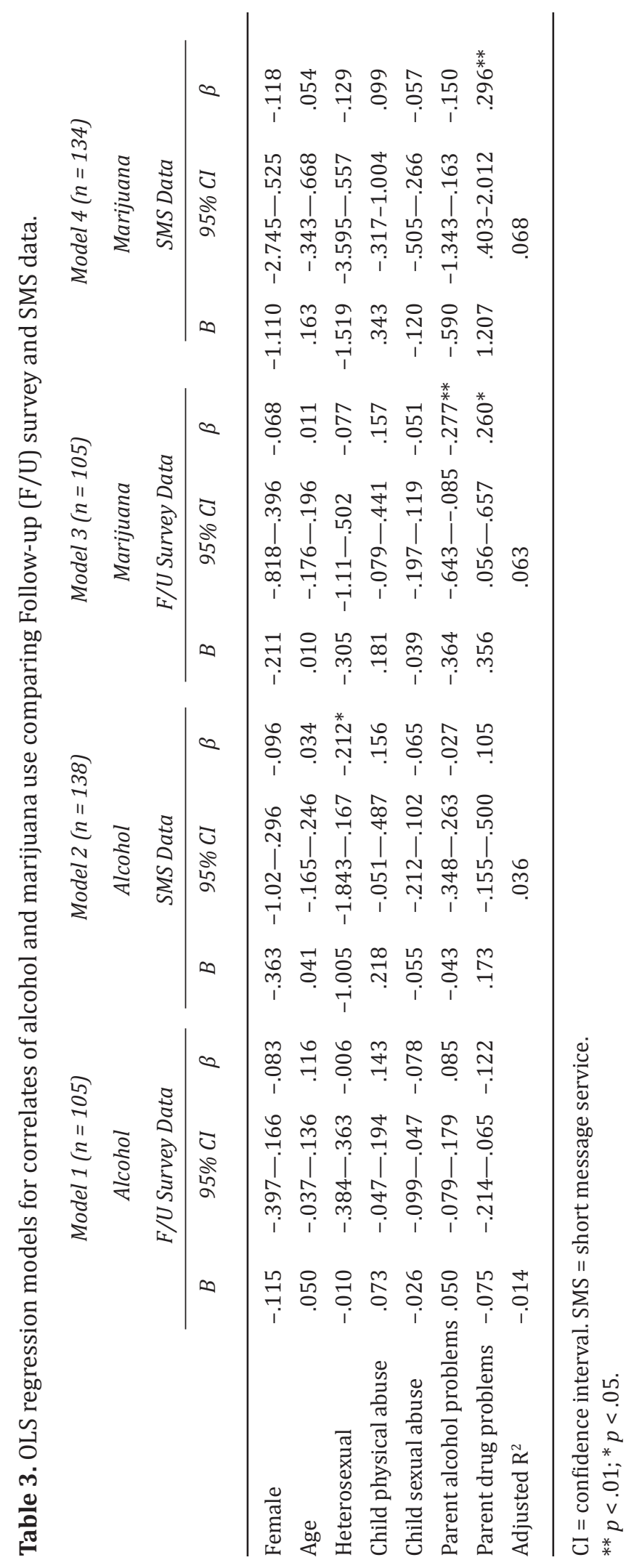


(see Models 1 and 2 ) revealed that the standardized coefficients ( $\beta$ ) between these two models were generally quite similar. There is one notable difference: heterosexual youth reported less alcohol use in the SMS data compared to the follow-up survey data $(\beta=-.212 ; p<.05$ vs. $\beta=$ $-.006 ; p>.05$, respectively).

For the marijuana use models (see Models 3 and 4) we also see similarities in the standardized coefficients across these two models. There is one notable difference: youth whose parents had alcohol problems reported less marijuana use in the survey data compared to the SMS data $(\beta=-.277 ; p<.01$ vs. $\beta=-.150 ; p>.05$, respectively).

\section{Discussion}

The purpose of our study was three-fold. First, we compared follow-up survey questionnaire data and SMS data to examine past 30-day alcohol use and past 30-day marijuana use. Second, we examined how similar actual reports of past 30-day ever drinking alcohol and ever using marijuana were for the follow-up survey and SMS data. Third, we examined whether demographic characteristics and early family risk factors were similarly associated with past 30-day alcohol and marijuana use for both follow-up survey questionnaire and SMS data.

Overall, comparing two different data collection techniques using the same time frame, we find that the association between past 30-day alcohol use comparing the follow-up survey and SMS data and past 30-day marijuana use using the same comparison yields moderately strong correlations. Moreover, when we compare "ever" versus "never" use, we find very similar reports comparing the SMS and follow-up survey data for alcohol use (42.4\% vs. 44.1\%, respectively) and for marijuana use $339.3 \%$ vs. $37.8 \%$, respectively) over the last 30 days. Neither substance was statistically significant from each other. These findings indicate that when querying homeless youth about ever drinking alcohol or ever using marijuana in the past 30 days, the use of either survey questionnaires or SMS surveying will attain very similar reliable results. However, given the additional benefits of using SMS for gathering data from homeless youth, such as being able to capture data "in the moment" about an individual's current behavior in their natural environment (9), the ability to verify the timing of one behavior relative to another, allowing for temporal 
sequencing (10) and minimizing recall biases (11), SMS provides many benefits beyond traditional survey methods. Additionally, SMS is wellsuited for use with a highly mobile population such as homeless youth.

Finally, we examined whether demographic characteristics and early family risk factors were similarly associated with past 30-day alcohol and marijuana use comparing follow-up survey and SMS data. Our results here show that demographic variables and family risk factors tend to be similarly associated with alcohol and marijuana use regardless of data collection method. In fact, we only find two notable differences, one each for alcohol and marijuana. These findings suggest that even when examining multivariate models, the variables are similarly associated with both outcomes and the strength of the coefficients are similar when comparing survey and SMS data. Thus, SMS, which is particularly wellsuited for use with homeless youth, may provide researchers with additional benefits beyond traditional survey questionnaires.

\section{Limitations}

In terms of limitations, although we have some information from youth across 2,768 youth-days, we are missing substance use data on 20\% of the youth-days, depending on the measure. Missing days of texting data is especially relevant when comparing the past 30 days survey reports with the SMS measures as many youth had completed the full 28 to 30 day time frame of texting. Second, the timing of the SMS question prompts captures the youth's experiences until that point but may have changed later that evening. For example, some youth may have used substances after the 9:30 pm set of questions, which would not be captured here. Similarly, substance use by youth earlier in the day also may not have been captured here. Third, although youth were asked about a variety of different types of illicit drugs, only marijuana had sufficient levels of reports to examine individually among this group of youth. It is possible that a longer study period may have yielded more drug use. Finally, our measure of past 30-day substance use asked the youth the number of times that they had used a substance, rather than the number of days on which a substance is used. If youth had used substances more than once per day, there may be discrepancies between the survey and SMS reports because of different scales in the items rather than differences in the accuracy of reporting or recall errors. 


\section{Conclusion}

Despite these limitations, our study has many strengths. The use of SMS surveying to collect daily data from homeless youth is relatively recent (Tyler \& Olson, 2018). As such, we know very little about the reliability of this method of data collection. Given that SMS surveying is well suited for highly mobile populations such as homeless youth, it is likely that we are going to see an increase in this type of data collection with this population. Thus, it is important to know if SMS is a reliable method. However, prior studies have yet to compare the reliability of survey questionnaires and SMS data on homeless youth's alcohol and marijuana use, which we have done here. Our results of this comparison reveal moderately strong correlations for alcohol and marijuana for past 30-day survey and SMS data. Additionally, results show that the reports between the SMS and survey follow-up data for "ever" using alcohol and marijuana are statistically equivalent. Moreover, results from multivariate models reveal that the associations between demographic characteristics and early family risk factors with alcohol and marijuana use are quite similar when comparing SMS and survey data suggesting that both are reliable methods of gathering data with this population when examining substance use. Thus, our study adds to the broader literature on substance use and homeless youth as our results indicate that SMS is also a reliable method for gathering substance use data from homeless youth. Given the numerous benefits of using SMS, it is likely we will continue to see a growth in this type of data collection when examining homeless youth populations in the future.

Disclosure The authors report no relevant financial conflicts.

Funding This article is based on research supported by a grant from the National Institute on Drug Abuse (DA036806). Dr. Kimberly A. Tyler, PI.

\section{References}

1. Kipke MD, Montgomery SB, MacKenzie RG. Substance use among youth seen at a community-based health clinic. J Adolesc Health. 1993;14:289-94. doi:10.1016/1054-139x(93)90176-p

2. Thompson SJ. Risk/protective factors associated with substance use among runaway/homeless youth utilizing emergency shelter services nationwide. Subst Abuse. 2004;25:13-26. 
3. Walls NE, Bell S. Correlates of engaging in survival sex among homeless youth and young adults. J Sex Res. 2011;48:423-36. doi:10.1080/00224499.2010.501916

4. Bousman CA, Blumberg EJ, Shilington AM, Hovell MF, Ji M, Lehman S, Clapp J. Predictors of substance use among homeless youth in San Diego. Addict Behav. 2005;30:1100-10. doi:10.1016/j.addbeh.2004.10.006

5. Wenzel SL, Tucker JS, Golinelli D, Green HD Jr., Zhou A. Personal network correlates of alcohol, cigarette, and marijuana use among homeless youth. Drug Alcohol Depend. 2010;112:140-49. doi:10.1016/j.drugalcdep.2010.06.004

6. Lim C, Rice E, Rhoades H. Depressive symptoms and their association with adverse environmental factors and substance use in runaway and homeless youths. J Res Adolesc. 2016;26:403-17. doi:10.1111/jora.12200

7. Santa MariaD, Padhye N, Yang Y, Gallardo K, Santao GM, Jung J, Businelle M. Drug use patterns and predictors among homeless youth: results of an ecological momentary assessment. Am J Drug Alcohol Abuse. 2017. doi:10.1080/00952990. 2017.1407328

8. Tyler KA, Olson K, Ray CM. Understanding the link between victimization and alcohol use among homeless youth using an ecological momentary assessment. Socius. 2018;4:1-7. Accessed June 6, 2018. doi:10.1177/2378023118779832

9. Shiffman S, Stone AA, Hufford MR. Ecological momentary assessment. Annu Rev Clin Psychol. 2008;4:1-32.

10. Cohn AM, Hunter-Reel D, Hagman BT, Mitchell J. Promoting behavior change from alcohol use through mobile technology: the future of ecological momentary assessment. Alcohol Clin Exp Res. 2011;35:2209-15. doi:10.1111/j.1530-0277.2011.01571.x

11. Kuntsche E, Labhart F. Using personal cell phones for ecological momentary assessment: an overview of current developments. Eur Psychol. 2013;18:3-11. doi:10.1027/1016-9040/a000127

12. Tyler K, Whitbeck LB. Lost childhoods: risk and resiliency among runaway and homeless adolescents. In: Allen-Meares P, Fraser MW, editors. Intervention with children and adolescents: an interdisciplinary perspective. Boston, MA: Pearson Education; 2004. p. 378-97.

13. Rowe C, Hern J, DeMartini A, Jennings D, Sommers M, Walker J, Santo GM. Concordance of text message ecological momentary assessment and retrospective survey data among substance-using men who have sex with men: A secondary analysis of a randomized controlled trial. JMIR MHealth Uhealth. 2016;4:e44. doi:10.2196/mhealth.5368

14. Tyler K. A., Olson K. Examining the Feasibility of Ecological Momentary Assessment Using Short Message Service Surveying with Homeless Youth: Lessons Learned. Field Methods 2018;30:91-104. Published Online First April 16, 2018. doi:10.1177/1525822X18762111

15. National Center for Homeless Education, The (NCHE) and the National Association for the Education of Homeless Children and Youth (NAEHCY). Updated 2017. Definitions of homelessness for federal programs serving children, youth, and families. Accessed February 24, 2018 https://nche.ed.gov/downloads/ briefs/introduction.pdf 
16. Ennett ST, Bailey SL, Federman EB. Social network characteristics associated with risky behaviors among runaway and homeless youth. J Health Soc Behav. 1999;40:63-78.

17. Straus MA, Hamby SL, Finkelhor D, Moore DW, Runyan D. Identification of child maltreatment with the Parent-Child Conflict Tactics Scales: development and psychometric data for a national sample of American parents. Child Abuse Negl. 1998;22:249-70. doi:10.1016/S0145-2134(97)00174-9

18. Whitbeck LB, Simons RL. Life on the streets: the victimization of runaway and homeless adolescents. Youth Soc. 1990;22:108-25. doi:10.1177/00441 18X90022001007

19. Tyler KA,Melander LA. Child abuse, street victimization, and substance use among homeless young adults. Youth Soc. 2015;47:502-19. doi:10.1177/0044118X12471354

20. Hodgins DC, Maticka-Tyndale E, El-Guebaly N, West M. The CAST-6: development of a short-form of the children of alcoholics screening test. Addict Behav. 1993;18:337-45. doi:10.1016/0306-4603(93)90035-8 